\title{
Self-cleaning of superhydrophobic nanostructured surfaces at low humidity enhanced by vertical electric field
}

\author{
Yijie Liu ${ }^{1}$, Yujun Guo ${ }^{1}(\bowtie)$, Xueqin Zhang ${ }^{1}$, Guoqiang Gao ${ }^{1}$, Chaoqun Shi ${ }^{1}$, Guizao Huang ${ }^{1}$, Pengli Li ${ }^{2}$, Qi Kang ${ }^{2}$, \\ Xingyi Huang ${ }^{2}(\triangle)$, and Guangning $\mathrm{Wu}^{1}(\Delta)$ \\ ${ }^{1}$ College of Electrical Engineering, Southwest Jiaotong University, Chengdu 611756, China \\ ${ }^{2}$ Department of Polymer Science and Engineering, Shanghai Key Laboratory of Electrical Insulation and Thermal Ageing, State Key laboratory of \\ Metal Matrix Composites, Shanghai Jiaotong University, Shanghai 200240, China
}

(C) Tsinghua University Press 2022

Received: 5 December 2021 / Revised: 17 December 2021 / Accepted: 21 December 2021

\section{ABSTRACT}

Self-cleaning is the key factor that makes superhydrophobic nanostructured materials have wide applications. The self-cleaning effect, however, strongly depends on formations and movement of water droplets on superhydrophobic nanostructured surfaces, which is greatly restricted at low humidity $\left(<7.6 \mathrm{~g}^{\mathrm{kg}} \mathrm{kg}^{-1}\right)$. Therefore, we propose a self-cleaning method at low humidity in which the pollution is electro-aggregated and driven in the electric field to achieve the aggregation and cleaning large areas. The cleaning efficiency of this method is much higher than that of water droplet roll-off, and will not produce "pollution bands". A simplified numerical model describing pollution movements is presented. Simulation results are consistent with experimental results. The proposed method realizes the self-cleaning of superhydrophobic nanostructured surfaces above dew point curve for the first time, which extends applications of superhydrophobic nanostructured materials in low humidity, and is expected to solve self-cleaning problems of outdoor objects in low humidity areas $\left(<5.0 \mathrm{~g} \cdot \mathrm{kg}^{-1}\right)$.

\section{KEYWORDS}

self-cleaning, electric field, low humidity, electro-aggregation, superhydrophobic nanostructured surface

\section{Introduction}

Objects exposed to air for a long time will be affected by particles in the atmosphere, resulting in serious surface pollution [1-4], which brings a series of adverse effects, such as solar power generation efficiency reduction $[5,6]$, and power equipment pollution flashover [7]. In the atmosphere, one type of particulate matter is from nature [8] (e.g., dust, soil, and sea salt), and the other is from human activities $[9,10]$ (e.g., burning soot and chemical particles). Self-cleaning, a significant feature of superhydrophobic nanostructured surfaces, can reduce surface pollution by providing the self-cleaning function to object surfaces [11-13]. This method has been widely used in the outdoor equipment of power transmission lines, the power supply equipment of high-speed railway [14], etc. However, the self-cleaning of superhydrophobic surfaces is extremely dependent on the movement of water droplets on the surface [15-17], which requires that the environmental conditions meet the dew point. This condition is difficult to achieve in low humidity arid areas, which restricts the application of superhydrophobic materials in deserts, Gobi, and other areas [18-20]. On the other hand, emerging energies such as solar and wind are often concentrated in these arid areas, which require the construction of many photovoltaic and wind power stations and the long-distance transmission of electricity to large cities and industrial areas. Realizing the surface self-cleaning of power equipment, wind power generation equipment, and energy transmission equipment can improve the power generation efficiency and ensure the operation of transmission lines.

Therefore, many efforts have been made to realize the self-cleaning property of superhydrophobic materials at low humidity. At present, the most common method is to change the environmental conditions to achieve the condensation of water droplets on the material surface. For example, by increasing humidity through artificial sprays [21], artificial rainfall [22,23], etc. and meeting the requirements of the environmental dew point, condensation on the material surface can be formed. Alternatively, one can condense water droplets on the material surface through a large temperature difference between day and night $[24,25]$, material cooling [26], and other methods. However, the volume of condensed water droplets produced by the above methods is too small, and the droplets more likely exist in the Wenzel state on the superhydrophobic material surfaces. In this case, the water droplets need a large sliding angle to move, which makes the selfcleaning efficiency extremely low.

An external field has been proposed to induce the directional motion of water droplets on the surface based on the theory of liquid dynamics. For example, ultrasonic waves [27], directional winds [28], and dip angles [29, 30] have been used so that water droplets on a surface experience a force, and directional movement occurs along the direction of the force. At the same time, temperature [31-33], pressure [34], and light intensity [35] gradients have also been studied to induce a change in water droplets on a superhydrophobic surface from the Wenzel state to 
the Cassie state. Then, the unstable water droplets on the surface fall off with the help of the inclination angle, thereby realizing self-cleaning. In addition, there are also studies to enhance the self-cleaning effect by electric field. These studies applied a horizontal electric force to the water droplets by the alternating current (AC)/direct current (DC) electric field, which can promote the movement of water droplets on the superhydrophobic surface and enhance the self-cleaning effect $[36,37]$. However, due to the small volume and the small number of water droplets on the surface, the cleaning effect of the above studies has a typical local feature, and multiple "clean bands" and "pollution bands" will appear on the material surface $[38,39]$, resulting in a low cleaning effect. Therefore, achieving efficient self-cleaning in low humidity remains a tremendous challenge. Although there are nearly 1,500 publications related to superhydrophobicity in 2020 alone, the self-cleaning method is still realized by the movement of water droplets, and the problem of low self-cleaning efficiency in low humidity has not been solved.

Here, we propose a self-cleaning method on a superhydrophobic nanostructured surface at low humidity with the help of electric field, as shown in Fig. 1. This method breaks away from the method of water droplets rolling and directly regulates the pollution, which can realize the self-aggregation and separation of pollution on a superhydrophobic nanostructured surface, and achieve a self-cleaning effect under low humidity conditions. In addition, the method avoids "pollution bands" that occur in the traditional method and achieves large-scale decontamination. This approach provides a new solution for the application of superhydrophobic materials in low humidity areas.

\section{Results and discussion}

\subsection{Fabrication and characterization of superhydro- phobic materials}

A superhydrophobic surface was prepared by using a mixture of fluoride-silane treated nano-silica with methyl silicone resin as a coating. Nano-silica was chosen because it is a commonly used functional filler for superhydrophobic coatings. Figure 2(a) shows a scanning electron microscopy (SEM) image of the coating.

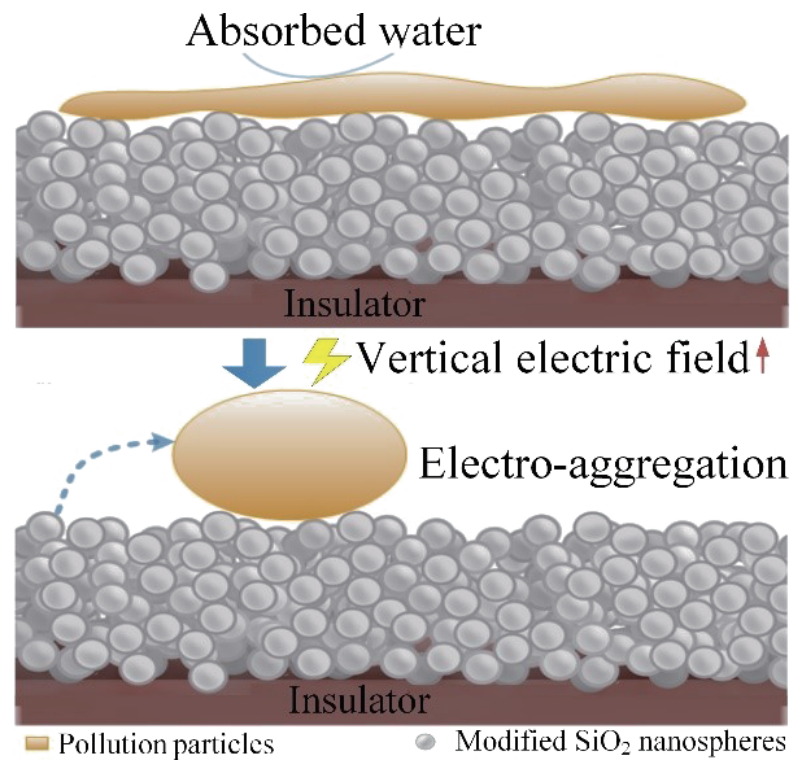

Figure 1 Self-aggregation of superhydrophobic surface pollution assisted by a vertical electric field. Pollution tiled on the superhydrophobic surface naturally absorbs moisture, and electro-aggregates under the action of a vertical electric field to form spherical pollutants.
Compared with a silicone rubber surface (Fig. 2(b)), the surface of the coating is irregularly distributed with bulges at the micron to submicron level, forming a micro/nano composite rough structure. The atomic force microscopy (AFM) results (Figs. 2(c) and 2(d)) show that the roughness of the coating (377 nm, Fig. S1 in the Electronic Supplementary Material (ESM)) is much higher than that of the silicone rubber substrate $(32.1 \mathrm{~nm}$, Fig. S2 in the ESM).

The roughness of micro/nanostructures is an important factor that can facilitate the low surface adhesion and high hydrophobicity of materials. The water droplet bounce test and surface static contact angle test further verify the results. As shown in Figs. 2(e) and 2(f) (Video ESM1), when a water droplet drops onto the material surface, it is difficult for the water droplet to adhere to the material surface, so it will rebound off. On the other hand, at $10{ }^{\circ} \mathrm{C}$ and a relative humidity of $60 \%$, the static contact angle of water on the coating surface is $158^{\circ}$. When the surface of the coating is covered with a layer of pollution (e.g., $\left.\mathrm{Fe}_{2}\left(\mathrm{SO}_{4}\right)_{3}\right)$, the surface is still hydrophobic with a static contact angle of $155^{\circ}$. This result indicates that pollution may have little effect on the superhydrophobicity of the coating (Fig. 2(g)).

The roughness of micro/nanostructures is an important factor that can facilitate the low surface adhesion and high hydrophobicity of materials. The water droplet bounce test and surface static contact angle test further verify the results. As shown in Figs. 2(e) and 2(f) (Video ESM1), when a water droplet drops onto the material surface, it is difficult for the water droplet to adhere to the material surface, so it will rebound off. On the other hand, at $10{ }^{\circ} \mathrm{C}$ and a relative humidity of $60 \%$, the static contact (a)

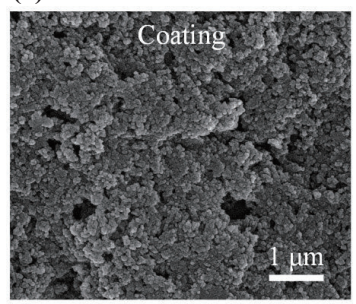

(c)

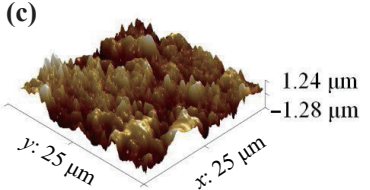

(b)

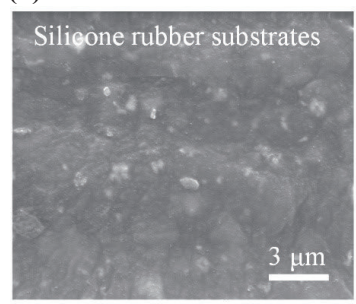

(d)

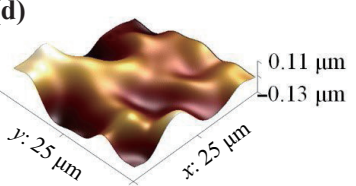

(e)
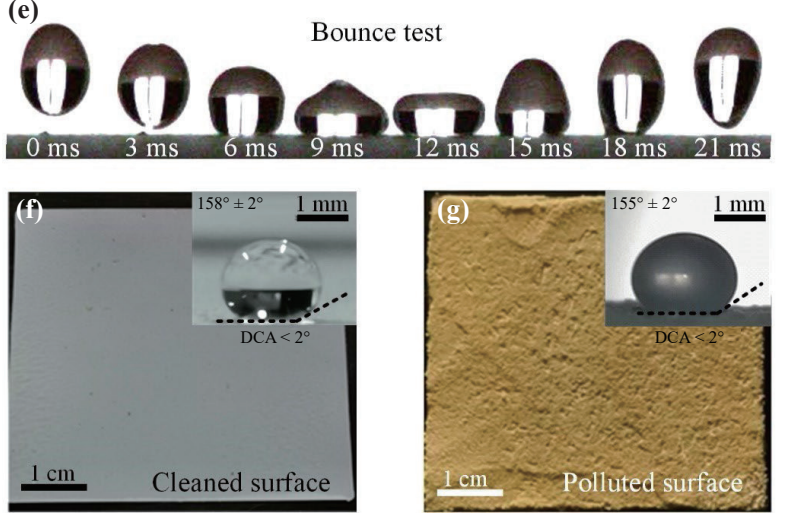

Figure 2 (a) SEM image of the coating surface, at a scale of $1 \mu \mathrm{m}$. (b) SEM image of a silicone rubber substrate surface, at a scale of $3 \mu \mathrm{m}$. (c) AFM image of the coating surface. (d) AFM image of the silicone rubber substrate surface. (e) Water droplet bounce test on the coating surface filmed by a high-speed camera. (f) Coating size and static contact angle of $158^{\circ} \pm 2^{\circ}$. The dynamic contact angle (DCA) is less than $2^{\circ}$. (g) Hydrophobicity test of the coating surface after spreading pollution. The static contact angle of the polluted surface is $155^{\circ} \pm 2^{\circ}$. The DCA is less than $2^{\circ}$. 
angle of water on the coating surface is $158^{\circ}$. When the surface of the coating is covered with a layer of pollution (e.g., $\left.\mathrm{Fe}_{2}\left(\mathrm{SO}_{4}\right)_{3}\right)$, the surface is still hydrophobic with a static contact angle of $155^{\circ}$. This result indicates that pollution may have little effect on the superhydrophobicity of the coating (Fig. $2(\mathrm{~g})$ ).

\subsection{Pollution self-aggregation results with the help of an electric field}

Figure 3(a) shows the self-aggregation behaviour of coating surface pollution in an electric field. When no electric field is applied, the pollution is evenly spread on the coating surface. After applying a $5 \mathrm{kV} \cdot \mathrm{cm}^{-1}$ electric field, the pollution accumulates towards the centre and bulges at the centre (Video ESM2). When the electric field intensity increases to $8 \mathrm{kV} \cdot \mathrm{cm}^{-1}$, the movement of the pollution concentration to the centre becomes faster, and thus the aggregation time is significantly shortened, as shown in Fig. 3(b). This finding indicates that in an electric field, the pollution will be electro-aggregated. The pollution contained water because of moisture absorption, and spherical mixtures can be formed because of the hydrophobicity of the superhydrophobic surface.

Figure 3(c) shows the effect of the electric field intensity on the pollution aggregation time. When the electric field intensity is less than $3.67 \mathrm{kV} \cdot \mathrm{cm}^{-1}$, the pollution will not spontaneously aggregate. When the electric field intensity exceeds $3.67 \mathrm{kV} \cdot \mathrm{cm}^{-1}$, the relationship between the electric field intensity and pollution aggregation time is inversely proportional. However, when the electric field intensity exceeds $30 \mathrm{kV} \cdot \mathrm{cm}^{-1}$, the air gap between the electrodes will breakdown and discharge. Additionally, the degree of pollution aggregation is also correlated with the environmental humidity. The greater the humidity is, the lower the electric field intensity is required by the aggregation effect (Fig. S3 in the ESM). With the help of an electric field, the self-aggregation of pollution on superhydrophobic surface can be realized at low humidity.

\subsection{Principle hypothesis of self-aggregation at low humidity}

To further explain the pollution self-aggregation behaviour at low humidity, a simplified model is proposed. At low humidity, the surface pollution, and water form solid-liquid coexistence. The adhesion force is far greater than the cohesion of water, and spontaneous aggregation is difficult, which is why the pollution is closely adhered to the material surface. According to the theory of electro-elasto-capillarity dynamics [40-42], the variation in solid-liquid coexistence on the material surface is related to the surface tension of the liquid and the elastic deformation parameters of the solid. When the pollution is in an electric field, the surface tension of the liquid tends to increase [43]. With the aid of the surface tension of the liquid, Maxwell stress, and vertical component of capillary action, the pollution is wrapped or adsorbed by the liquid, and forms a sphere under the hydrophobic effect of the superhydrophobic materials to achieve spontaneous aggregation, as shown in Fig. 4(a) (for more details see the Note S2 in the ESM). The simplified model can represent the relationship between the electric field intensity and the pollution aggregation time, and its relationship is as follows

$$
t=\sqrt{\frac{2 \Delta S \rho}{\left(k_{1} E^{2}+k_{2}\right)}}\left(\frac{\partial \overrightarrow{\boldsymbol{m}}}{\partial \varphi}\right)^{-2}
$$

where $t$ is the aggregation time, $\Delta S$ is the change in the surface distance, $\rho$ is the density of pollution, $E$ is the electric field intensity, $k_{1}$ and $k_{2}$ are the fitting coefficients, and their values are related to material properties and environmental states, $\varphi$ is the phase field variables, and $\overrightarrow{\boldsymbol{m}}$ is the unit vector in the direction of the resultant force.

\subsection{Simulation results}

The above pollution aggregation model at low humidity was used for simulation analysis (Simulation parameters are shown in Table S2 in the ESM). In the simulation, when no electric field is applied, the pollution is tiled on the coating surface. In electric fields of 5 and $8 \mathrm{kV} \cdot \mathrm{cm}^{-1}$, the pollution aggregates into a small ball, and the aggregation times are 14 and $4 \mathrm{~s}$, respectively. The results are consistent with the experimental results, as shown in Figs. 3(a) and 3(b). Moreover, the curve of the electric field intensity versus aggregation time is shown in Fig. 3(c). Compared with the experimental results, the maximum relative error is $13.02 \%$ (Tables S3 and S4 in the ESM). Through the above test and simulation results, it can be seen that the pollution can aggregate into a ball on the surface of superhydrophobic materials in an electric field, and the aggregation time is inversely proportional to the electric field intensity.

\subsection{The self-cleaning result of aggregation pollution}

The removal of pollution is an important step for the self-cleaning of superhydrophobic surfaces, which can be realized by further increasing the electric field intensity. This is because when the electric field force of pollution exceeds its own gravity and the surface adhesion force, it will break away from the superhydrophobic surface. However, at low humidity, the pollution (ferric sulfate) is in a solid-liquid mixture state, showing an anisotropic and uneven distribution. The above model only considers the continuous and uniform distribution of pollution and cannot truly simulate the pollution movement behaviour. For this reason, pseudo-random sampling is used to simulate the anisotropic and uneven distribution of the pollution, and the
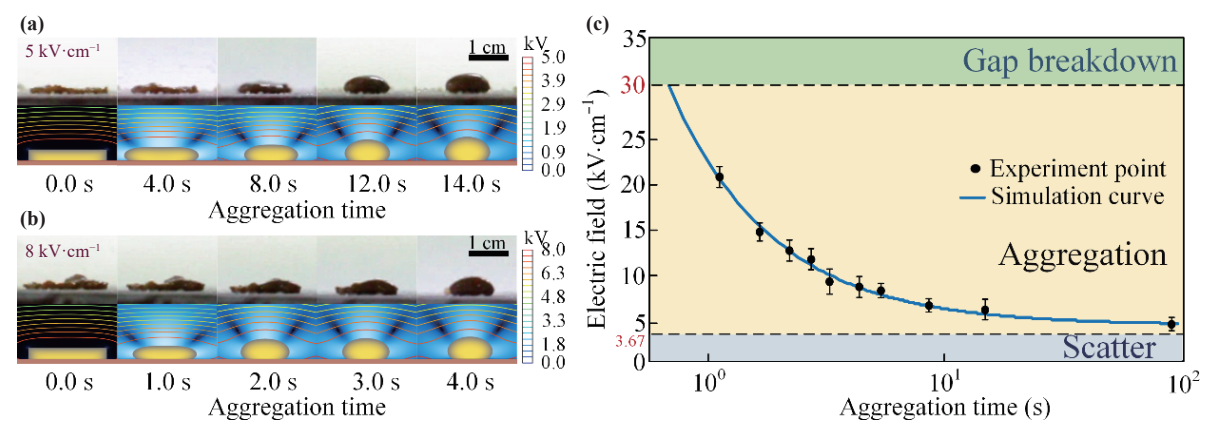

Figure 3 Characteristics of the electro-aggregation of pollution. (a) Pollution aggregation process at $5 \mathrm{kV} \cdot \mathrm{cm}^{-1}$ and a total time of $14 \mathrm{~s}$. (b) Pollution aggregation process at $8 \mathrm{kV} \cdot \mathrm{cm}^{-1}$ and a total time of $4 \mathrm{~s}$. For (a) and (b), the above image is the test result and the following image is the simulation result. (c) Curve of the electric field intensity and aggregation time. The black dots represent the test data points and the blue curve represents the simulation results. At humidity $5.0 \mathrm{~g} \cdot \mathrm{kg}^{-1}$, the minimum field intensity required for pollution aggregation is $3.67 \mathrm{kV} \cdot \mathrm{cm}^{-1}$. 
(a)
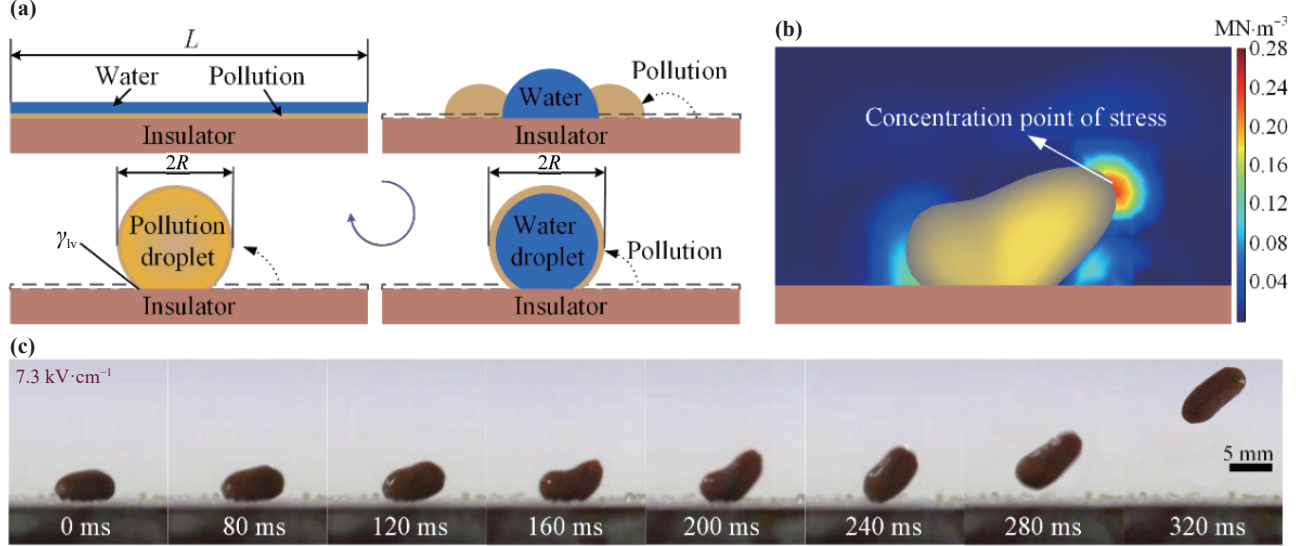

(d)

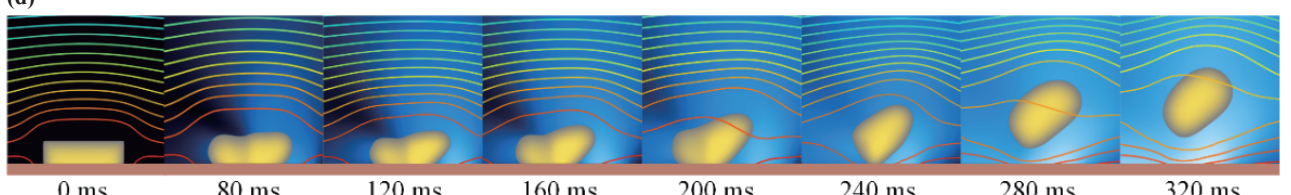

(e)

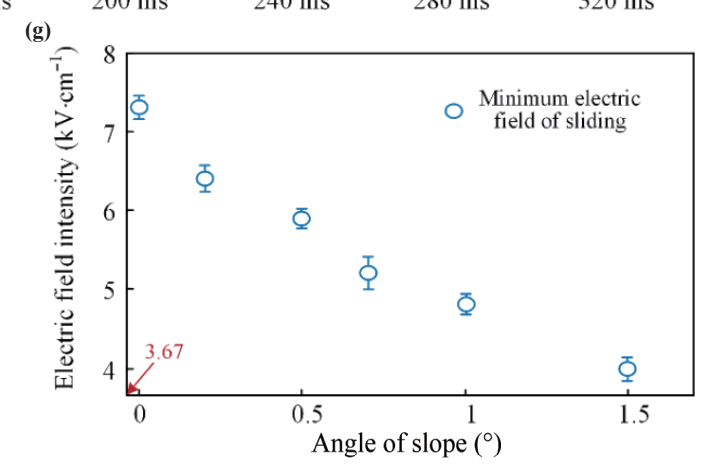

Figure 4 Characteristics of self-cleaning under electro fluid mechanics. (a) Schematic diagram of the critical conditions for the spontaneous aggregation of pollution. The blue part is the water film formed by pollution absorbing water. The yellow part is the pollution. The red part is the silicone rubber substrate. Under the action of an electric field, the pollution aggregates with the help of moisture absorption to form a spherical mixture. (b) The characteristics of the volume force on the pollution surface simulated at $7.3 \mathrm{kV} \cdot \mathrm{cm}^{-1}$. In this case, stress concentration exists in the bulge, and the maximum volume force is $0.28 \mathrm{mN} \cdot \mathrm{m}^{-3}$. (c) The test process of pollution take-off and self-cleaning at $7.3 \mathrm{kV} \cdot \mathrm{cm}^{-1}$. The total time is $320 \mathrm{~ms}$. (d) Simulation process of pollution take-off and self-cleaning at $7.3 \mathrm{kV} \cdot \mathrm{cm}^{-1}$. The total time is $320 \mathrm{~ms}$. (e) The test process of pollution sliding down the inclined plane under $4 \mathrm{kV} \cdot \mathrm{cm}^{-1}$, the slip angle is $1.5^{\circ}$ and the total time is $9.1 \mathrm{~s}$. (f) Simulation process of pollution sliding down the inclined plane under $4 \mathrm{kV} \cdot \mathrm{cm}^{-1}$, the slip angle is $1.5^{\circ}$ and the total time is $9.1 \mathrm{~s}$. $(\mathrm{g})$ The relationship between the minimum electric field intensity and the inclination angle of the pollution sliding from the inclined plane under the condition of pollution aggregation.

results are shown in Figs. 4(b)-4(d). When only an electric field is applied, the aggregation speeds of the left and right sides of pollution are inconsistent, and there is an initial momentum to the right. Local convex spots will appear in the area with a slow contraction. In the electric field environment, local convex points will lead to an electric field distortion (Fig. 4(b)), which further causes changes in the Maxwell stress and Coulomb force, thus aggravating the development of contaminated surface bulges. The stress concentration will occur in pollution bumps, leading to tensile development along with the bumps. At the same time, the pollution is a solid-liquid mixture, with a large viscosity and Young's modulus. The force will develop along the tensile direction of the jump, and the overall pollution breaks away from the material surface to realize the self-cleaning of the surface.

To further verify the process of pollution take-off, the pollution (ferric sulfate) on the surface is wetted at $10{ }^{\circ} \mathrm{C}$ to increase its weight by $0.05 \mathrm{~g}$ and remains in a solid-liquid mixing state (Fig. S5 in the ESM). It is found in the test (Fig. 4(c)) that with increasing electric field intensity, the pollution first jumps off and then escapes from the superhydrophobic surface (Video ESM3), and the test results are consistent with the simulation results. At $120 \mathrm{~ms}$, the pollution is anisotropic and the surface is unevenly stressed, forming a bulge in the upper right corner. The bulge further leads to an electric field distortion, which intensifies the polarization effect, and causes the pollution to stretch and jump along the bulge direction. Finally, the pollution breaks away from the superhydrophobic surface.

When the electric field intensity exceeded $7.3 \mathrm{kV} \cdot \mathrm{cm}^{-1}$, the pollution could jump from the surface of the superhydrophobic material. That is, the electric field intensity required for pollution separation is far greater than that required for self-aggregation, which is not conducive for practical applications. Therefore, a surface inclination angle can be introduced to reduce the electric field intensity of pollution movement. In fact, the advantage of an inclination angle has been widely utilized in actual engineering (e.g., photovoltaic panels and insulator sheds). In Fig. 4(e), when the surface inclination angle is $1.5^{\circ}$, the electric field intensity of pollution sliding is reduced to $4 \mathrm{kV} \cdot \mathrm{cm}^{-1}$ (Video ESM4). Moreover, the sliding process is simulated and analyzed by the above model. Figure 4(f) shows that the pollution starts to slide slowly, and the rate increases with time. This result indicates that sliding detachment can be achieved in a low electric field with the help of the inclination angle, and the electric field intensity required by pollution sliding decreases with increasing inclination angle (Fig. $4(\mathrm{~g})$ ). With the aid of the inclination angle, the pollution motion is changed from jumping to sliding, and the required electric field intensity is significantly reduced. Therefore, the slope surface can be used to achieve the self-cleaning of superhydrophobic surfaces at a low electric field intensity. 


\section{Practical application at low humidity}

\subsection{Self-aggregation results of different pollutants at low humidity}

In practical engineering applications, there are many kinds of pollution. For example, for overhead transmission equipment and solar power generation equipment, the most common pollution includes sodium chloride, calcium sulfate, ammonium sulfate, diatomite, kaolin, and dust. We selected the above six types of pollution to carry out the experiment shown in Fig. 5(a). Under unwetted conditions, the pollution is completely tiled on the surface. Wetting in a humidity of $5.7 \mathrm{~g} \mathrm{~kg}^{-1}$ and a temperature of $10{ }^{\circ} \mathrm{C}$, the pollution edge condenses inward, but all of the pollution is still tiled on the surface. When an electric field is introduced, the pollution aggregates towards the centre, forming a spherical mixture of liquid and solid. Further analysis of the aggregation characteristics of the different pollutants showed that diatomite had the most obvious aggregation characteristics (Fig. S6 in the ESM). The above tests also show that the common pollution on the surface of the overhead transmission equipment can be aggregated by an electric field at low humidity. This finding indicates that although pollution aggregation is closely related to the material properties, the electric field-assisted aggregation

(a)

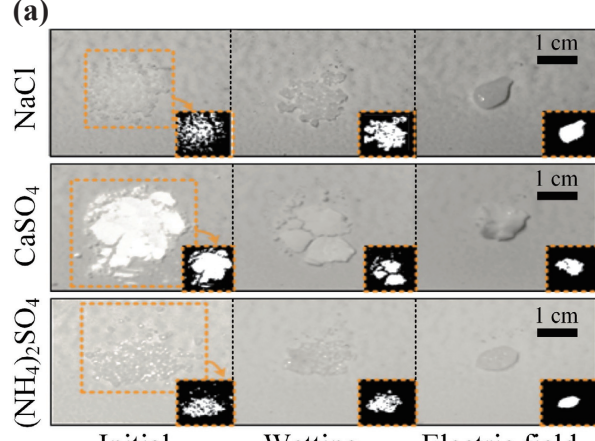

(b)
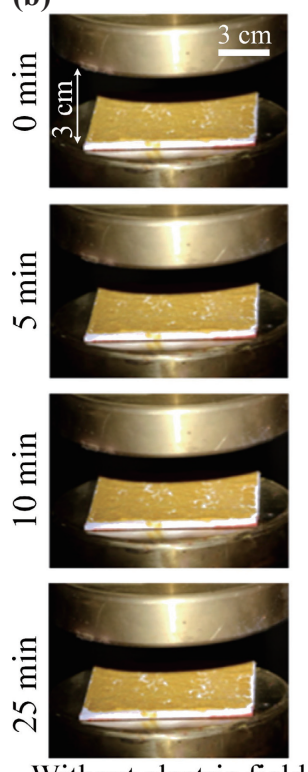

Without electric field

Wetting Electric field
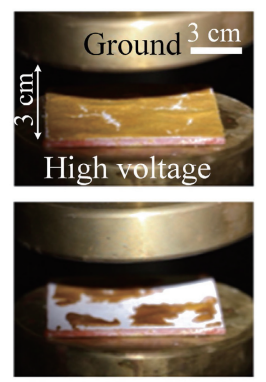

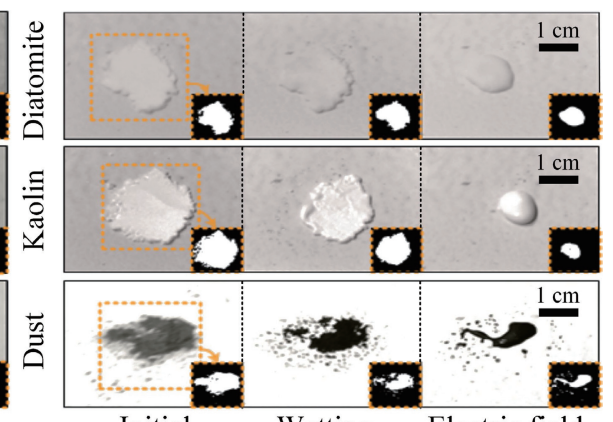

(c)

behaviour at low humidity is universal.

\subsection{Self-cleaning effect of the overall pollution in a large} area

In practical engineering, pollution usually exists on the overall surface of the material. Therefore, a self-cleaning characteristic test was also conducted on a contaminated surface (Fig. 5(b)). The two groups of pollution with the same mass were wetted in an environment of $5.0 \mathrm{~g} \cdot \mathrm{kg}^{-1}$ humidity and $10{ }^{\circ} \mathrm{C}$, and removed after the same weight gain. For the group without electric field, the pollution did not change after long standing. However, in the group with an electric field of $5 \mathrm{kV} \cdot \mathrm{cm}^{-1}$, there was a clear boundary between the pollution aggregation, forming multiple concentration areas. In the aggregation area, the pollution forms a solid-liquid mixed sphere, which will slide down an inclined plane in an electric field. In other words, at low humidity, self-cleaning with the help of an electric field can realize the overall self-cleaning of a large area of pollution. In addition, we also compare our new self-cleaning method with traditional self-cleaning by water droplets at low humidity. As shown in Fig. 5(c), in the case of a small number of water droplets, water droplets can only remove the pollution in the falling area, showing significant local characteristics. However, our new self-cleaning method can aggregate and then remove the whole pollution, which can
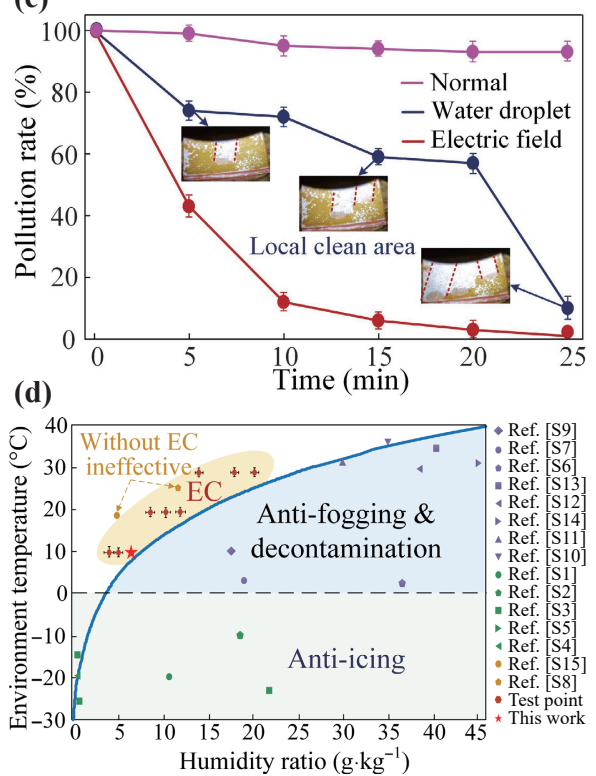

Figure 5 Practical application of self-cleaning with the help of an electric field. (a) The aggregation characteristics of different pollutants. (b) Self-cleaning effect of overall pollution in a large area. The inclination angle in the test was $1.5^{\circ}$. The applied electric field intensity is $4 \mathrm{kV} \cdot \mathrm{cm}^{-1}$. (c) Comparison of the decontamination efficiency of without an electric field, with an electric field, and with water droplets. The water droplets fell on the material surface at three times of 0 , 10, and 20 min. (d) Comparison of the environmental conditions of self-cleaning on superhydrophobic surfaces reported in the literature with the results in this paper. The curve is a dew point curve, below which condensation can spontaneously form water droplets on the material surface. Green dots indicate good anti-icing performance, and purple dots indicate the application category of superhydrophobicity under traditional conditions. The yellow spot indicates that it is difficult to form water droplets on the surface in this environment, and it is difficult to realize self-cleaning without the influence of external factors. 
remove all pollution in a large area under low humidity (Video ESM5).

The dew point curve is the boundary curve of water condensation in the air. The environmental conditions required for traditional self-cleaning should be below the dew point curve. As shown in Fig. 5(d), and Table S1 and Note S1 in the ESM, the self-cleaning environment of superhydrophobic surfaces reported at present is mainly concentrated in the area below the dew point curve. However, we realized self-cleaning in the environment above the dew point curve and expanded the application of superhydrophobic materials in low humidity environments, including desert, drought, and other areas in the environment above the dew point curve for a long time.

\section{Conclusions}

In conclusion, a new self-cleaning method at low humidity on superhydrophobic surfaces is proposed. This method utilizes the electro-elasto-capillarity deformation and cohesion characteristics of pollution in an electric field and uses the changes in the Maxwell stress and surface tension on the surface to realize pollution aggregation, jumping, and breaking away from the surface. This method can also reduce the electric field intensity required for pollution movement by using the surface inclination angle, and realizing the self-cleaning of the surface in a low electric field. Compared with the traditional self-cleaning method (i.e., water droplets roll away pollution), the electric field-assisted method can achieve the self-cleaning of pollution in a large area, which is more practical in engineering applications. Moreover, we realized the self-cleaning of superhydrophobic surfaces above the dew point curve for the first time with the assistance of an electric field, and expanded the application of superhydrophobic materials in low humidity environments, such as desert and drought areas.

\section{Methods}

\subsection{Materials}

Perfluorodecyl trimethoxysilane copolymer (PTC, Guangzhou Hongcheng Biotechnology Co., Ltd., China) and hydrophobic vapour phase nano-silica particles (15-30 nm in diameter, Zhitai Purification Technology Co., Ltd., China) were obtained commercially and used as received. Ethyl alcohol (Yantai Songyu Trading Co., Ltd., China) and methyl silicone resin (Hubei Xinsihai Chemical Industry Co., Ltd., China) were used as solvents and film formers, respectively. A commercial product silicone rubber insulator $(5 \mathrm{~cm} \times 5 \mathrm{~cm} \times 0.5 \mathrm{~cm}$, Wenzhou Yikun electric Co., Ltd., China) was chosen as the substrate. Ferric sulfate powders $\left(\mathrm{Fe}_{2}\left(\mathrm{SO}_{4}\right)_{3}\right.$, Zhiyuan Chemical Reagent Co., Ltd., China) were obtained as the pollution. In addition, all other chemicals were analytical-grade reagents and were used as received.

\subsection{Fabrication of the super hydrophobic surface}

The preparation procedure to prepare the superhydrophobic surface mainly consisted of three steps. The first step was the preparation of a precursor nanocomposite suspension. PTC $(5 \mathrm{~mL})$ was diluted with ethyl alcohol $(25 \mathrm{~mL})$ and the solution was stirred at $200 \mathrm{r} \cdot \mathrm{min}^{-1}$ and at room temperature for $10 \mathrm{~min}$. Meanwhile, hydrophobic vapour phase nano-silica particles were dispersed in ethyl alcohol $(25 \mathrm{~mL})$ and ultrasonicated for $10 \mathrm{~min}$. Then the PTC solution and silica nanoparticle dispersion were mixed, and deionized water was dripped at $1 \mathrm{~mL} \cdot \mathrm{min}^{-1}$. The mixed solution was ultrasonically dispersed with water bath heating $\left(60{ }^{\circ} \mathrm{C}\right)$ for $3 \mathrm{~h}$ to obtain a homodisperse nanocomposite suspension. The second step was the preparation of the superhydrophobic surface. The silica-PTC suspension $(50 \mathrm{~mL})$ and methyl silicone resin $(0.67 \mathrm{~g})$ were ultrasonically dispersed for $30 \mathrm{~min}$. Moreover, silicone rubber substrates were cleaned with deionized water and dried. Afterwards, the silica-PTC mixed solution was spray deposited onto the silicone rubber substrates by an airbrush with a nozzle diameter and compressed air pressure of $1.5 \mathrm{~mm}$ and $0.29 \mathrm{MPa}$, respectively. The third step was crosslinking and curing at high temperature. The samples were kept at $120{ }^{\circ} \mathrm{C}$ for $2 \mathrm{~h}$ to crosslink and remove the residual solvents. Finally, the superhydrophobic surface was fabricated. The water contact angles (WCAs) of the samples were measured with a contact angle metre (CAM, Xiangtan Huafeng Instrument Manufacturing Co., Ltd., China) by the sessile drop method.

\subsection{Pollution movement experiment process assisted by an electric field}

Two circular plate electrodes (T2 pure copper) were used for the test. The diameter and thickness of the electrode were 7 and $1.5 \mathrm{~cm}$, respectively. The space of the two electrodes was $3 \mathrm{~cm}$. The bottom electrode and the high-voltage DC generator (ZGF-120 kV/5 mA) were connected, and the top electrode was connected to the ground. A uniform electric field was created between the electrodes. Before the test, the samples were cleaned by anhydrous alcohol. After drying the samples were covered with a layer of ferric sulfate powder. Finally, the samples were placed in an artificial fog chamber to be affected by damping. When the weight of the samples increased $0.05 \mathrm{~g}$, the samples were moved and placed between the electrodes. The test circuit is shown in Fig. S6 in the ESM. A camera (Canon EOS 700D, Japan) and a highspeed camera (FASTCAM Nova S6, Japan) were used to record the movement behaviour of damp pollution in a constant direct current electric field.

\section{Acknowledgements}

The authors are grateful to the supporting program of the National Natural Science Foundation of China (Nos. 51837009 and 51907168).

Electronic Supplementary Material: Supplementary material (experimental procedures, computational details, modeling process, supplementary figures, tables, and videos) is available in the online version of this article at https://doi.org/10.1007/s12274022-4093-0.

\section{References}

[1] Feng, L.; Li, S.; Li, Y.; Li, H.; Zhang, L.; Zhai, J.; Song, Y.; Liu, B.; Jiang, L.; Zhu, D. Super-hydrophobic surfaces: From natural to artificial. Adv. Mater. 2002, 14, 1857-1860.

[2] Gao, X. F.; Jiang, L. Water-repellent legs of water striders. Nature 2004, 432, 36.

[3] Oh, J.; Dana, C. E.; Hong, S.; Román, J. K; Jo, K. D.; Hong, J. W.; Nguyen, J.; Cropek, D. M.; Alleyne, M.; Miljkovic, N. Exploring the role of habitat on the wettability of cicada wings. ACS Appl. Mater. Interfaces 2017, 9, 27173-27184.

[4] Mouterde, T.; Lehoucq, G.; Xavier, S.; Checco, A.; Black, C. T.; Rahman, A.; Midavaine, T.; Clanet, C.; Quéré, D. Antifogging abilities of model nanotextures. Nat. Mater. 2017, 16, 658-663.

[5] Wang, D. H.; Sun, Q. Q.; Hokkanen, M. J.; Zhang, C. L.; Lin, F. Y.; Liu, Q.; Zhu, S. P.; Zhou, T. F.; Chang, Q.; He, B. et al. Design of robust superhydrophobic surfaces. Nature 2020, 582, 55-59.

[6] Geng, Y.; Jiao, K.; Liu, X.; Ying, P. J.; Odunmbaku, O.; Zhang, Y. X.; Tan, S. C.; Li, L.; Zhang, W.; Li, M. Applications of bioderived/bio-inspired materials in the field of interfacial solar steam generation. Nano Res., in press, https://doi.org/10.1007/s12274-021- 
3834-9.

[7] Li, Y. F.; Jin, H. Y.; Nie, S. C.; Zhang, P.; Gao, N. K. Dynamic behavior of water droplets and flashover characteristics on a superhydrophobic silicone rubber surface. Appl. Phys. Lett. 2017, 110, 201602.

[8] Escher, B. I.; Stapleton, H. M.; Schymanski, E. L. Tracking complex mixtures of chemicals in our changing environment. Science 2020, 367, 388-392.

[9] Le, T. H.; Wang, Y.; Liu, L.; Yang, J. N.; Yung, Y. L.; Li, G. H.; Seinfeld, J. H. Unexpected air pollution with marked emission reductions during the COVID-19 outbreak in China. Science 2020, 369, 702-706.

[10] Lin, Z. Z.; Wang, Z.; Zhang, X.; Diao, D. F. Superhydrophobic, photo-sterilize, and reusable mask based on graphene nanosheetembedded carbon (GNEC) film. Nano Res. 2021, 14, 1110-1115.

[11] Lu, Y.; Sthasivam, S.; Song, J. L.; Crick, C. R.; Carmalt, C. J.; Parkin, I. P. Robust self-cleaning surfaces that function when exposed to either air or oil. Science 2015, 347, 1132-1135.

[12] Chen, Y. C.; Huang, Z. S.; Yang, H. T. Cicada-wing-inspired selfcleaning antireflection coatings on polymer substrates. ACS Appl. Mater. Interfaces 2015, 7, 25495-25505.

[13] Zhang, L. L.; Meng, G.; Fan, G. F.; Chen, K. L.; Wu, Y. L.; Liu, J. High flux photocatalytic self-cleaning nanosheet $\mathrm{C}_{3} \mathrm{~N}_{4}$ membrane supported by cellulose nanofibers for dye wastewater purification. Nano Res. 2021, 14, 2568-2573.

[14] Sun, Q. Q.; Wang, D. H.; Li, Y. N.; Zhang, J. H.; Ye, S. J.; Cui, J. X.; Chen, L. Q.; Wang, Z. K.; Butt, H. J.; Vollmer, D. et al. Surface charge printing for programmed droplet transport. Nat. Mater. 2019 18, 936-941.

[15] Zuo, Y. X.; Zheng, L. Z.; Zhao, C.; Liu, H. Micro-/Nanostructured interface for liquid manipulation and its applications. Small 2020, 16, 1903849.

[16] Barthlott, W.; Neinhuis, C. Purity of the sacred lotus, or escape from contamination in biological surfaces. Planta 1997, 202, 1-8.

[17] Wang, R.; Wu, F. F.; Yu, F. F.; Zhu, J.; Gao, X. F.; Jiang, L. Antivapor-penetration and condensate microdrop self-transport of superhydrophobic oblique nanowire surface under high subcooling. Nano Res. 2021, 14, 1429-1434

[18] Zhang, J.; Zhu, C. X.; Lv, J.; Zhang, W. C.; Feng, J. Preparation of colorful, infrared-reflective, and superhydrophobic polymer films with obvious resistance to dust deposition. ACS Appl. Mater. Interfaces 2018, 10, 40219-40227.

[19] Rao, A. V.; Latthe, S. S.; Mahadik, S. A.; Kappenstein, C. Mechanically stable and corrosion resistant superhydrophobic solgel coatings on copper substrate. Appl. Surf. Sci. 2011, 257, $5772-5776$.

[20] Bumbudsanpharoke, N.; Ko, S. Nanomaterial-based optical indicators: Promise, opportunities, and challenges in the development of colorimetric systems for intelligent packaging. Nano Res. 2019, 12, 489-500.

[21] Jadhav, A. J.; Holkar, C. R.; Pinjari, D. V. Anticorrosive performance of super-hydrophobic imidazole encapsulated hollow zinc phosphate nanoparticles on mild steel. Prog. Org. Coat. 2018, $114,33-39$.

[22] Wang, L.; Yang, J. Y.; Zhu, Y.; Li, Z. H.; Sheng, T.; Hu, Y. M.; Yang D. Q. A study of the mechanical and chemical durability of ultra-ever dry superhydrophobic coating on low carbon steel surface. Coll. Surf. A Physicochem. Eng. Aspect. 2016, 497, 16-27.

[23] Zhu, C. X.; Lin, W. Q.; Chen, L. D.; Lv, J.; Zhang, J.; Feng, J. Deep color, heat-reflective, superhydrophobic and anti-soiling coatings with waterborne silicone emulsion. Sol. Energy Mater. Sol. Cells 2019, 199, 129-135.
[24] Goswami, D.; Medda, S. K.; De, G. Superhydrophobic films on glass surface derived from trimethylsilanized silica gel nanoparticles. ACS Appl. Mater. Interfaces 2011, 3, 3440-3447.

[25] Gauthier, A.; Symon, S.; Clanet, C.; Quéré, D. Water impacting on superhydrophobic macrotextures. Nat. Commun. 2015, 6, 8001.

[26] Adera, S.; Raj, R.; Enright, R.; Wang, E. N. Non-wetting droplets on hot superhydrophilic surfaces. Nat. Commun. 2013, 4, 2518.

[27] Liu, M. J.; Wang, S. T.; Jiang, L. Nature-inspired superwettability systems. Nat. Rev. Mater. 2017, 2, 17036.

[28] Yao, C. W.; Lai, C. L.; Alvarado, J. L.; Zhou, J.; Aung, K. T.; Mejia, J. E. Experimental study on effect of surface vibration on micro textured surfaces with hydrophobic and hydrophilic materials. Appl. Surf. Sci. 2017, 412, 45-51.

[29] Hassan, G.; Yilbas, B. S.; Al-Sharafi, A.; Al-Qahtani, H. Selfcleaning of a hydrophobic surface by a rolling water droplet. Sci. Rep. 2019, 9, 5744 .

[30] Li, J.; Jing, Z. J.; Zha, F.; Yang, Y. X.; Wang, Q. T.; Lei, Z. Q. Facile spray-coating process for the fabrication of tunable adhesive superhydrophobic surfaces with heterogeneous chemical compositions used for selective transportation of microdroplets with different volumes. ACS Appl. Mater. Interfaces 2014, 6, 8868-8877.

[31] Seo, D.; Lee, J.; Lee, C.; Nam, Y. The effects of surface wettability on the fog and dew moisture harvesting performance on tubular surfaces. Sci. Rep. 2016, 6, 24276.

[32] Choo, S.; Choi, H. J.; Lee, H. Water-collecting behaviour of nanostructured surfaces with special wettability. Appl. Surf. Sci. 2015, 324, 563-568.

[33] Wang, N.; Xiong, D. S. Superhydrophobic membranes on metal substrate and their corrosion protection in different corrosive media. Appl. Surf. Sci. 2014, 305, 603-608.

[34] Lambley, H.; Schutzius, T. M.; Poulikakos, D. Superhydrophobic surfaces for extreme environmental conditions. Proc. Natl. Acad. Sci. USA 2020, 117, 27188-27194

[35] Li, W.; Tang, X.; Wang, L. Q. Photopyroelectric microfluidics. Sci. $A d v . \mathbf{2 0 2 0}, 6,1693$.

[36] Ren, S.; Wang, S. W.; Dong, Z. Q.; Chen, J.; W. Li, L. E. Dynamic behaviors and self-cleaning property of droplet on superhydrophobic coating in uniform DC electric field. Colloids Surf. A Physicochem. Eng. Aspect. 2021, 626, 127056.

[37] Li, J.; Wei, Y.; Huang, Z. Y.; Wang, F. P; Yan, X. Z.; Wu, Z. L. Electrohydrodynamic behavior of water droplets on a horizontal super hydrophobic surface and its self-cleaning application. Appl. Surf. Sci. 2017, 403, 133-140.

[38] Qing, Y.; Shi, S. L.; Lv, C. J.; Zheng, Q. S. Microskeleton-nanofiller composite with mechanical super-robust superhydrophobicity against abrasion and impact. Adv. Funct. Mater. 2020, 30, 1910665.

[39] Lee A.; Moon, M. W.; Lim, H.; Kim, W. D.; Kim, H. Y. Water harvest via dewing. Langmuir 2012, 28, 10183-10191.

[40] Py, C.; Reverdy, P.; Doppler, L.; Bico, J.; Roman, B.; Baroud, C. N. Capillary origami: Spontaneous wrapping of a droplet with an elastic sheet. Phys. Rev. Lett. 2007, 98, 156103.

[41] Zhao, Y. B. Physical Mechanics of Surface and Interface; Science Press: Beijing, China, 2012.

[42] Liu, Y. J.; Wu, G. N.; Guo, Y. J.; Zhang, X. Q.; Liu, K.; Kang, Y. Q.; Shi, C. Q. Pollution agglomeration characteristics on insulator and its effect mechanism in DC electric field. Int. J. Elec. Power Energy Syst. 2020, 115, 105447.

[43] Gibbons, M. J.; Garivalis, A. I.; O’Shaughnessy, S.; Di Marco, P.; Robinson, A. J. Evaporating hydrophilic and superhydrophobic droplets in electric fields. Int. J. Heat Mass Transfer 2021, 164, 120539. 